experimental lupus model and the effect of ER stress inhibition by 4-PBA on the frequency and function of Treg.

Methods: Murine lupus model were induced with female BALB/c mice at 7 or 8 weeks of age through Toll-like receptor (TLR) agonist 7 treatment for 4 weeks. From the 8th week, the mice were treated with phosphate buffered saline (PBS), 4-PBA (500 mg/kg, three times weekly) and dexamethasone $(1 \mathrm{mg} / \mathrm{kg}$, once a day) for 4 weeks. The increment of body weight, spleen weight, anti-doublestranded DNA (anti-dsDNA) antibody titer, serum cytokine level and the pathology of glomerulonephritis were analysed at 12 weeks of age. The population of immune cellular subset including activated T, B lymphocyte and Treg and suppressive functions of Treg were measured.

Results: 4-PBA significantly decreased the level of anti-dsDNA antibodies, serum TNF- $\alpha$ in murine lupus model, and which were comparable with the efficacy of dexamethasone. A significant decrease in accumulation of immunoglobulin, glomerulonephritis score was also observed in 4-PBA-treated and dexamethasone-treated mice compared with vehicle-treated group. ER stress inhibition decreased the activated $\mathrm{T}$ and $\mathrm{B}$ lymphocytes population of splenocytes, but the population of Treg was not significantly different between vehicle group and 4PBA group. However, there was the markedly enhanced suppressive capacity of Treg in 4-PBA-treated group.
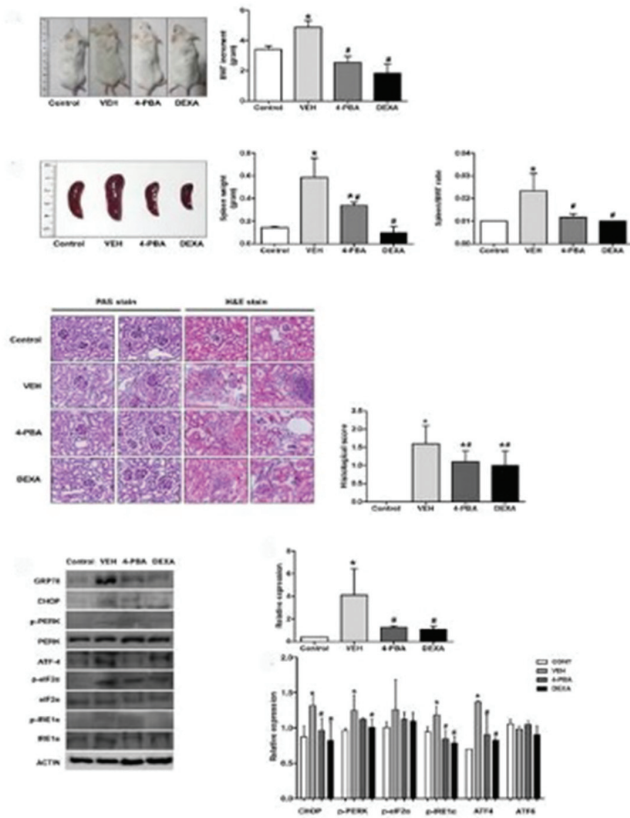

Abstract AB0178 - Figure 1

Conclusions: The results suggest that ER stress inhibition attenuates disease activity in experimental model, especially in nephritis through improving the suppressive capacity of Treg. Thus, reduction of ER stress could be used as a beneficial therapeutic strategy in SLE.

Disclosure of Interest: None declared

DOI: 10.1136/annrheumdis-2018-eular.2879

\section{AB0179 SERUM LEVELS OF INTERLEUKIN-33 ARE ASSOCIATED WITH DEPRESSIVE SYMPTOMS IN PATIENTS WITH PRIMARY SJÖGREN'S SYNDROME}

Z. $\mathrm{Li}^{1}, \mathrm{~T} . \mathrm{Fu}^{1}, \mathrm{C} . \mathrm{Dong}^{1}$, L. Li ${ }^{2}$, Z. Gu${ }^{1} .{ }^{1}$ Department of Rheumatology, Affiliated Hospital of Nantong University; ${ }^{2}$ Department of Gastroenterology, Affiliated Hospital of Nantong University, Nantong, P.R. China., Nantong, China

Background: Primary Sjögren's syndrome (pSS) is a systemic autoimmune disease which is more common among women than men. Extraglandular manifestations are commonly reported, including central nervous system (CNS) involvement such as headaches, cognitive deficits and mood disorder. Among mood disorder, depression is one of the most frequent disorders observed in patients with pSS. The pathogenesis of depression in pSS remains unclear.

Objectives: The aim of this study was to explore potential relationships between serum interleukin-33 (IL-33) levels and depressive symptoms in primary Sjögren's syndrome (pSS) patients.
Methods: We included 116 consecutive pSS patients (36 depression patients), and 71 (18 depression patients) age-and sex-matched healthy controls. The presence of depressive symptoms was determined through the Hospital Depression Scale (HDS). IL-33 levels were measured by enzyme-linked immunosorbent assay (ELASA) using commercial kits.

Results: There was no significant difference between pSS patients and controls. But, in pSS patients, the serum IL-33 levels were significantly lower in depression patients as compared to patients without depression [40.18 (IQR, 31.79$66.70) \mathrm{ng} / \mathrm{ml}$ vs. 39.95(IQR, 121.73) $\mathrm{ng} / \mathrm{ml} ; \mathrm{Z}=-3.029, \mathrm{p}=0.002$ ]. A direct negative correlation between the score of HDAS-D and sera IL-33 levels $(r=-0.805$ $\mathrm{p}=0.000$ ) in pSS was also observed. In logistic regression model using depression as the dependent variables, IL-33 is the independent predictors of depression in pSS $(\beta=0.004 ; \mathrm{OR}=1.004 ; 95 \% \mathrm{Cl}: 1.998-0.011 ; \mathrm{p}=0.170)$. Surprisingly, sera IL33 levels were lower in depression patients with pSS compared to depression patients without $\mathrm{pSS}(\mathrm{Z}=-2.316, \mathrm{p}=0.021)$.

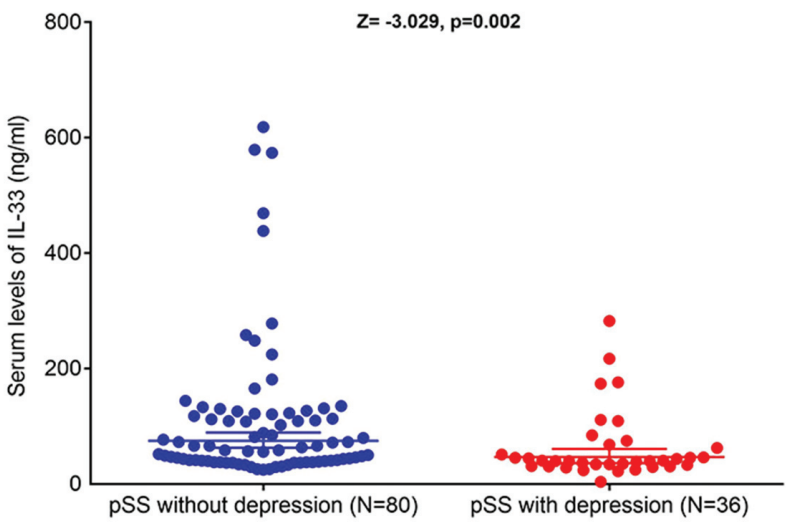

Abstract AB0179 - Figure 1

Conclusions: Conclusion: In pSS, IL-33 is the independent predictors of depressive disorders. Serum IL-33 levels are decreased in pSS patients with depressive symptoms, suggesting that IL-33 may play a unique role in depression with pSS.

Acknowledgements: This study was funded by the Chinese National Natural Science Foundation (Grant no. 81671616, and 81471603); Jiangsu Provincia Commission of Health and Family Planning Foundation (Grant no. H201317 and H201623); Science Foundation of Nantong City (Grant no. MS32015021, MS2201564, MS22016028 and MS22016019); Science and Technology Foundation of Nantong City (Grant no. HS2014071 and HS2016003).

Disclosure of Interest: None declared

DOI: 10.1136/annrheumdis-2018-eular.6110

\section{$\mathrm{AB} 0180$ \\ IDENTIFICATION OF LNCRNA LNCMKLN1 CONTRIBUTED TO ABNORMAL ACTIVATION OF TYPE I INTERFERON PATHWAY IN SYSTEMIC LUPUS ERYTHEMATOSUS}

Z. Ye, Y. Tang, Z. Yin, X. Chen, Y. Chen. Shenzhen Futian Hospital for Rheumatic Diseases, Shenzhen, China

Background: Dysregulation or dysfunction of some key moleculars in signalling pathway is involved in disease pathogenesis. Type I interferon pathway abnormal activation has been identified as major molecular phenotype of lupus patients. Long non-coding RNA (IncRNA), as a regulator of gene expression, plays great role in signalling pathway.

Objectives: We hypothesised that dysregulation of IncRNA may involve in key signalling pathway of SLE.

Methods: Deep sequencing of human renal samples to screen differential expression of IncRNAs between LN patients and healthy donors. RNA-FISH was used to identify subcellular location of IncRNA. Stimulation in human renal mesangial cells (HRMC) by all kinds of TLR ligands, IFNs, and TNF $\alpha$, and transfection in HRMC cells by antisense oligonucleotides (ASOs), and quantitative real-time polymerase chain reaction (RT-qPCR), enzyme linked immunosorbent assay (ELISA) were used to analyse the relative genes expression. LncMKLN1 transcription was activated or inhibited through CRISPR-dCas9 system in Hela cell line. RNA-seq was executed to examine the gene expression profile after changing IncMKLN1 expression, and western blot was applied to determine the key signalling molecules of IFN pathway. 